Olivier Burgy ${ }^{1,15}$, Jesse Armitage ${ }^{2,3,15}$, Louise Wain ${ }^{4,5,15}$, Maribel Casas $6,7,8,15$, Leandro Cruz Mantoani ${ }^{9,15}$, Elsa Bodier-Montagutelli10,11,12,15, Cristina Boccabella ${ }^{13,15}$, Jana De Brandt ${ }^{14}$

\title{
ERS International Congress 2018: highlights from best-abstract awardees
}

\section{Introduction}

During the 2018 European Respiratory Society (ERS) International Congress in Paris the author of the best abstract from every assembly was awarded (based on the highest average score from the abstract reviewers for an author under 40 years of age). Best-abstract awardees were invited to write a short summary of their Congress experience and view on the evolving field of research of their respective assembly. The purpose of this article is to give the readers a fast overview of some of the Congress highlights and to give the stage to the young and promising best-abstract awardees, as they are the future of the ERS.

\section{Assembly 3: Basic and translational sciences (Olivier Burgy)}

One of the highlights of the 2018 ERS International Congress was a seminal symposium on disease modelling and drug testing focusing on innovative three-dimensional models using human lung biosamples to study lung diseases (organoids and lung tissue culture). In addition, the recent ERS statement on optimising the use of animal models in respiratory diseases was thoroughly discussed [1-3]. The importance of Wnt/ $\beta$-catenin signalling in lung epithelial cell homeostasis and response to lung injury was presented in a hot topic session on "Pathophysiology of the human lung alveolus". William J. Zacharias (Cincinnati, OH, USA) shared exciting recent findings about a specific subpopulation of alveolar epithelial progenitor cells [4]. Cellular crosstalk, specifically novel mediators such as extracellular vesicles and exosomes, represented another highlight. Susanne Gabrielsson (Stockholm, Sweden) presented exosomes as potential biomarkers in human lung diseases. She demonstrated that bronchoalveolar lavage fluid (BALF)-derived exosomes from sarcoidosis patients carry higher levels of vitamin-D binding protein compared with controls [5]. Exosomes from sarcoidosis patients have leukotriene-dependent pro-inflammatory properties and trigger interleukin- $1 \beta$ production in macrophages. Hannah Burke (Southampton, UK) and co-workers showed that exosomes have dysregulated microRNA (miRNA) relevant to inflammatory pathways and might predict chronic obstructive pulmonary disease (COPD) [6]. Olivier Burgy (Denver, CO, USA) and co-workers demonstrated an increase of extracellular vesicles in BALF from patients with idiopathic pulmonary fibrosis (IPF) [7]. They showed that BALF-extracellular vesicles from fibrotic mice exhibit a specific proteome compared to non-fibrotic mice. In line with this, Julien Guiot (Liège, Belgium) and co-workers shared data on a specific miRNA signature in sputum-exosomes from patients with IPF [8]. They found that the miRNA miR-142-3p is increased in IPF exosomes and correlates with disease severity. Together, these sessions emphasised the knowledge and proof of concept generated by our community with the aim

@ERSpublications

A summary of the ERS International Congress 2018 from the best-abstract awardees for each ERS Assembly and their views on the evolving field of research of their respective Assemblies http://ow.ly/c0eq30ntKuw

Cite as: Burgy O, Armitage J, Wain L, et al. ERS International Congress 2018: highlights from best-abstract awardees. Breathe 2018; 14: e137-e142. 
of providing better therapies for those suffering from lung diseases.

\section{Assembly 5: Airway diseases, asthma and COPD (Jesse Armitage)}

The airways diseases Assembly showcased many interesting presentations ranging from strategies to develop and improve clinical practice for COPD and asthma patients, to understanding the pathological mechanisms that drive these diseases. Both COPD and asthma exhibit a broad spectrum of features, some of which overlap significantly. Consequently, understanding what clinical features these patients present remains a crucial factor in providing positive clinical outcomes in response to therapy. This was emphasised during the symposium "State of the art session: airways diseases", which presented the importance of delivering therapeutics that are tailored to the patients' clinical features. For example, Dave Singh (Manchester, UK) highlighted the association between higher blood eosinophil levels in COPD patients and favourable responses to inhaled corticosteroids $[9,10]$. The variety of work undertaken towards the development of novel therapeutic strategies for both COPD and asthma was particularly impressive. Several approaches were explored including the use of stem cells, monoclonal antibodies, antibiotics and other immunomodulators. One such study, presented by Peter Gibson (Newcastle, Australia) demonstrated that macrolides, a class of antibiotics, can have numerous anti-inflammatory and antimicrobial effects that can benefit patients with neutrophilic COPD and poorly controlled asthma [11, 12]. Other presentations in this assembly also focused on unravelling the mechanisms that drive these complex airway diseases. The sessions "It's my favourite cell that affects your asthma" and "Advanced profiling of immune cells in chronic lung diseases" were great examples of this, as they displayed a breadth of talks emphasising the role of immune cell subsets and extracellular vesicles in airways diseases and how these findings can be translated clinically as biomarkers and therapeutic targets. Collectively, this serves as a reminder of the enormous challenges ahead when addressing these complex diseases, and the requirement to approach the treatment of airways diseases with new perspectives.

\section{Assembly 6: Epidemiology and environment (Louise Wain)}

It is an exciting time for genomic studies in respiratory disease. Evidence that genetic data can accelerate and improve drug discovery pipelines [13], with increasing recognition of the potential for genomics to revolutionise patient care [14], has catalysed the field of genetic studies. The potential benefits, and challenges, of undertaking genetic studies for fine-scale respiratory phenotypes were explored in a thought-provoking "Multiomics studies in epidemiology: what can they tell us?" session. Elizabeth Oelsner (New York, USA) demonstrated how we can take genetic risk scores (where multiple individual genetic risk factors are summed into a single score for each individual) derived from large general population studies of spirometry lung function and COPD risk to assess their association with computed tomography scan measures. The associations of such risk scores with lung structure provide new mechanistic insights [15]. Natalie Terzikhan (Rotterdam, the Netherlands) quantified the heritability of diffusing capacity and reported that a G-protein-coupled-receptor, associated with airflow obstruction, was also associated with measurements of gas exchange. This suggest a role for this protein in alveolar function [16]. Epidemiological studies provide unequivocal evidence that our exposure to airborne pollutants is an important driver of respiratory health. However, our understanding of how these exposures lead to lung disease, and how environmental impact varies across the population, is unknown. The challenges of addressing these questions were showcased in the "Gene-environment interactions in the omics era" symposium by leaders in the field of gene-byenvironment studies. Florence Demenais (Paris, France) provided recommendations for optimal study designs to maximise statistical power to identify and replicate gene-by-environment interactions [17]. Erik Melén (Stockholm, Sweden) made the case for integration of multiple "omics" data to fortify findings with functional evidence. Ian P. Hall (Nottingham, UK) described how the effects of genetic risk factors for COPD were independent of the already large relative risk from smoking. In the "multiomics" session, Louise Wain (Leicester, UK) demonstrated that, amongst smokers, the absolute risk of COPD by age 70 varies from around $17 \%$ to $84 \%$ according to genetic risk profile [18]. In summary, these sessions highlighted the value of deeply phenotyped studies and the potential of multiomics studies to provide new insight into respiratory health and disease.

\section{Assembly 7: Paediatrics (Maribel Casas)}

At the 2018 ERS International Congress, more than 350 studies focusing on the field of paediatrics were presented. Clinicians and researchers emphasised the need to consider lung function trajectories in future asthma guidelines to better monitor lung growth throughout life based on two papers recently published in the Lancet Respiratory Medicine [19, 20]. Regarding bronchopulmonary dysplasia (BPD), recent studies showed that intrauterine growth restriction, maternal smoking and pre-existing hypertension 
were associated with increased risk of BPD [21] and that BPD impairs cognitive development and quality of life in infants born extremely preterm [22]. Concerning the early-life origins of respiratory disorders, two cohorts showed that school-aged asthma was influenced by earlier lower respiratory tract infections or asthma symptoms, but not by upper respiratory tract infections [23, 24]. A study in more than 200000 dyads observed that maternal antibiotic use during pregnancy was associated with a dose-dependent increase in asthma risk during childhood [25]. In the symposium dedicated to the early-origins of respiratory disorders, researchers discussed the different approaches to strengthen causal inference. This included the approach of gene-environment interactions, such as a study that examined the influence of a particular variant of the glutathione S-transferase gene on the association between paracetamol use during the first years of life and the risk of developing asthma in early adulthood [26]. Other approaches discussed were family designs such as sibling controls, twin studies and negative parental controls, and studies following children initially involved in randomised controlled trials [27]. Studies focusing on environmental exposures such as air pollution [28], green spaces [29] or pesticides [30] and their potential effects on respiratory disorders were highly represented at the Congress. Novel studies on the influence of the internal (i.e. metabolome) and external exposome [31, 32] on respiratory health were also presented. In conclusion, the 2018 ERS International Congress covered a broad spectrum of respiratory diseases including new insights into potential underlying mechanisms, treatment and prevention of respiratory diseases presented by an exciting mix of international experts in the field.

\section{Assembly 9: Allied respiratory professionals (Leandro Cruz Mantoani)}

The 2018 ERS International Congress was full of great topics. For the members of Assembly 9, there were two very interesting postgraduate courses to kick off the conference: "PG8 Lung function testing", providing an update on the current techniques for measuring lung physiology; and "PG14 The evolving world of pulmonary rehabilitation", which provided a better understanding of the role of pulmonary rehabilitation (PR) in the integrated continuous care of patients with chronic lung diseases. The excellent symposium on "Dyspnoea: a multidimensional and multidisciplinary approach" discussed the many aspects dyspnoea can have, topics included: the neurophysiology of dyspnoea, exercise and dyspnoea, the multidimensional assessment of dyspnoea, and the clinical impact and management of dyspnoea. Another outstanding session was the oral presentation session: "Determinants of physical activity behaviour in patients with COPD and the physiotherapist as a physical activity coach". Speakers from all over the world brought new insights into physical activity behaviour in COPD, highlighting the role of the physiotherapist as a coach to encourage COPD patients to achieve higher levels of daily physical activity. The oral presentation session "Best abstracts in pulmonary rehabilitation and chronic care" showed that PR is a continuously evolving field of research: researchers brought findings from "Early PR after acute exacerbation of COPD" [33] to "Regular extended release of morphine for chronic breathlessness" [34]. The poster discussion about "What's new in lung function and exercise assessment in COPD?" caught the attention of many people as well. This session involved some hot topics in the field, with researchers investigating the "Impact of $\mathrm{FEV}_{1}$ / FVC threshold to predict COPD hospitalisation and mortality" [35] and the "Relationship between four-meter gait speed test and cardiopulmonary exercise test to determine exercise capacity in COPD patients" [36], among others. Overall, the 2018 ERS International Congress provided insights into new topics, with thought-provoking sessions highlighting the importance of treating and assessing the patient thoroughly.

\section{Assembly 10: Respiratory infections (Elsa Bodier-Montagutelli)}

Within the area of basic and translational research, a large number of sessions were dedicated to the role of the microbiome in lung pathophysiology. Philip Hansbro (Callaghan, Australia) provided insight into how regulation of the microbiome and cross-talk with the lung plays a fundamental role in homeostasis with his presentation on "Gut-lung cross-talk in chronic respiratory disease". In addition, evidence was presented for the association of certain microbiome patterns, including the gut microbiome, with chronic respiratory diseases (especially in early childhood) or clinical response to immunotherapies in lung cancer. Understanding the role of the microbiome also opens innovative therapeutic perspectives: Patricia Lepage (Jouy-en-Josas, France) discussed microbiome manipulation or specific bacterial metabolites as options for the prevention or treatment of chronic respiratory diseases during her presentation on "Manipulating the microbiome to control human disease". The development of therapeutic alternatives was also mentioned for respiratory infections, with a special focus on bacterial resistance to antibiotics. The prevalence and extent of drug resistance was assessed in many descriptive studies covering various infectious lung diseases, notably lower respiratory tract infections and tuberculosis, which are two of the five respiratory diseases with the biggest impact [37]. In this context, 
antimicrobial resistance was one of the priority concerns of the "10 Principles for Lung Health" presented at the Congress and recently published by the ERS [38]. Several potential alternatives to antibiotics were discussed during the symposium on "Antimicrobial host defence and innate immunity in the lung", including antimicrobial peptides, therapeutic antibodies and phages [39]. Multidrug resistance to tuberculosis was also highlighted during the Congress in a thematic poster session on "Drug-resistant tuberculosis and the sequelae of pulmonary tuberculosis" and a poster discussion session on "Drug-resistant tuberculosis". A stateof-the-art session was provided, combining current practical recommendations and the challenges that remain to reach the eradication of disease. Advances were also presented on the comprehension of bronchiectasis pathophysiology, as well as epidemiological microbiological data. Overall, the 2018 ERS International Congress highlighted the key interactions between basic, translational and clinical science in the field of respiratory infections, opening new perspectives for understanding disease pathogenesis, prevention and treatment.

\section{Assembly 12: Interstitial lung diseases (Cristina Boccabella)}

Interstitial lung diseases (ILDs) are a heterogeneous group of disorders characterised by an infiltrative lung process of the interstitium. ERS selected this topic as one of the eight key educational fields for the ERS International Congress 2018, in view of the recent growing interest among researchers and the large amount of scientific literature produced. The new guidelines for diagnosing IPF [40], published in September 2018 in the American Journal of Respiratory and Critical Care Medicine were discussed during a highly interactive plenary session. Chairs and speakers described in detail the methodology used to develop the guidelines and the implications for clinical practice. Different to the 2011 guidelines, four radiological categories were defined: usual interstitial pneumonia (UIP), probable UIP, indeterminate or inconsistent for UIP pattern. As in the 2018 Consensus Statement of the Fleischner Society, the possible UIP pattern was excluded from the classification. Bronchoalveolar lavage and surgical lung biopsy received a conditional indication to be performed after multidisciplinary team discussion, according to radiological and clinical evaluations. Possible limitations of the new recommended approach could be the subjective interpretation of radiological patterns and the need for experts. Furthermore, Toby Maher (St. Albans, UK) presented the INMARK study (NCT02788474) [41], a 12-week, double blind, randomised, placebo controlled, parallel group, ongoing trial that aims to evaluate the effects of Nintedanib on a broad panel of biomarkers of extracellular matrix turnover. This study will hopefully provide support in identifying predictive biomarkers of IPF clinical course and new targets for antifibrotic therapy. Finally, the INSTAGE study [42], a 24-week, randomised, double-blind, parallel-group trial, recently published in the New England Journal of Medicine, assessed the efficacy and safety of Nintedanib in addition to Sildenafil in IPF patients. Although the first end-point was negative in terms of improvements in quality of life, the INSTAGE study, as Fernando J. Martinez (New York, USA) highlighted, could provide new insights on patients with severely impaired gas exchange, potentially supporting the use of Nintedanib across a wider range of IPF patients. The ILD Assembly gave an overview on the new diagnostic approaches for IPF, highlighting their possible implications and challenges in clinical practice. Future perspectives were shared about the use of antifibrotic treatment and the potential role of IPF-specific biomarkers.

\section{Concluding remarks}

We hope to reach out to and inspire all young clinicians and researchers to submit an abstract for the ERS International Congress 2019, in Madrid, as this gives them an opportunity to be elected as the 2019 best-abstract awardee from their Assembly. Abstract submission is open until February 14, 2019.

\footnotetext{
Affiliations

Olivier Burgy 1,15, Jesse Armitage 2,3,15, Louise Wain 4,5,15, Maribel Casas $6,7,8,15$, Leandro Cruz Mantoani ${ }^{9,15}$, Elsa Bodier-Montagutelli ${ }^{10,11,12,15}$, Cristina Boccabella ${ }^{13,15}$, Jana De Brandt ${ }^{14}$

1Division of Pulmonary Sciences and Critical Care Medicine, Dept of Medicine, University of Colorado Denver, Aurora, CO, USA. ${ }^{2}$ Centre for Respiratory Health, School of Biomedical Sciences, University of Western Australia, Perth, Australia. ${ }^{3}$ Stem Cell Unit, Institute of Respiratory Health, Perth, Australia. ${ }^{4}$ Dept of Health Sciences, University of Leicester, Leicester, UK. ${ }^{5}$ National Institute for Health Research, Leicester Respiratory Biomedical Research Centre, Glenfield Hospital, Leicester, UK. ${ }^{\circ}$ SSGlobal, Barcelona, Spain. ${ }^{7}$ CIBER Epidemiología y Salud Pública (CIBERESP), Barcelona, Spain. ${ }^{8}$ Universitat Pompeu Fabra, Barcelona, Spain. ${ }^{9} \mathrm{CEPPOS}-$ Centre of Research and Post-graduation, Associated Postgraduation Programme in Rehabilitation Sciences, State University of Londrina and University of the North of Paraná, Londrina, Brazil. ${ }^{10}$ Centre d'Etude des Pathologies Respiratoires, Université de Tours, Tours, France. ${ }^{11}$ Centre d'Etude des Pathologies Respiratoires, U1100, INSERM, Tours, France. ${ }^{12}$ CHRU de Tours, Service de Pharmacie, Tours, France. ${ }^{13}$ Cardiovascular and Thoracic Dept, Piliclinico "A. Gemelli", IRCCS, University of the Sacred Heart, Rome, Italy. ${ }^{14}$ REVAL - Rehabilitation Research Center, BIOMED - Biomedical Research Institute, Faculty of Rehabilitation Sciences, Hasselt University, Diepenbeek, Belgium. ${ }^{15}$ These authors contributed equally
} 


\section{Conflict of interest}

O. Burgy has nothing to disclose. J. Armitage has nothing to disclose. L. Wain reports grants from GSK, outside the submitted work. M. Casas has nothing to disclose. L.C. Mantoani reports grants and nonfinancial support from Philips Research Eindhoven, outside the submitted work. E. Bodier-Montagutelli has nothing to disclose. C. Boccabella has nothing to disclose. J. De Brandt has nothing to disclose.

\section{Support statement}

J. De Brandt is funded by the Flemish government. The Research of FWO Aspirant J. De Brandt is sponsored by FWO-grant \#11B4718N. O. Burgy is supported by a postdoctoral fellowship from the European Respiratory Society and the European Molecular Biology Organization (ERS/EMBO Joint Research Fellowship - Nr. LTRF 2016-7481).

\section{References}

1. Alsafadi HN, Staab-Weijnitz CA, Lehmann M, et al. An ex vivo model to induce early fibrosis-like changes in human precisioncut lung slices. Am J Physiol Lung Cell Mol Physiol 2017; 312: L896-L902.

2. Bonniaud P, Fabre A, Frossard N, et al. Optimising experimental research in respiratory diseases: an ERS statement. Eur Respir J 2018; 51: 1702133.

3. Nikolic MZ, Caritg O, Jeng Q, et al. Human embryonic lung epithelial tips are multipotent progenitors that can be expanded in vitro as long-term self-renewing organoids. Elife 2017; 6: e26575.

4. Zacharias WJ, Frank DB, Zepp JA, et al. Regeneration of the lung alveolus by an evolutionarily conserved epithelial progenitor. Nature 2018; 555: 251-255.

5. Martinez-Bravo MJ, Wahlund CJ, Qazi KR, et al. Pulmonary sarcoidosis is associated with exosomal vitamin D-binding protein and inflammatory molecules. J Allergy Clin Immunol 2017; 139: 1186-1194

6. Burke $\mathrm{H}$, Heinson A, Freeman A, et al. Late Breaking Abstract - Differentially expressed exosomal miRNAs target key inflammatory pathways in COPD. Eur Respir J 2018; 52: Suppl. 62, OA4922.

7. Martin-Medina A, Lehmann M, Burgy O, et al. Increased extracellular vesicles mediate wnt-5a signaling in idiopathic pulmonary fibrosis. Am J Respir Crit Care Med 2018; in press [https://doi.org/10.1164/rccm.201708-15800C].

8. Njock MS, Guiot J, Henket MA, et al. Sputum exosomes: promising biomarkers for idiopathic pulmonary fibrosis. Thorax 2018; in press [https://doi.org/10.1136/ thoraxjnl-2018-211897].

9. Pascoe S, Barnes N, Brusselle G, et al. Late Breaking Abstract - Blood eosinophil counts and treatment response in COPD: analyses of IMPACT. Eur RespirJ 2018; 52: Suppl. 62, OA2127.

10. Agusti A, Fabbri LM, Singh D, et al. Inhaled corticosteroids in COPD: friend or foe? Eur Respir J 2018; 52 : 1801219

11. Baines KJ, Wright TK, Gibson PG, et al. Azithromycin treatment modifies airway and blood gene expression networks in neutrophilic COPD. ERJ Open Res 2018; 4: 00031-2018.

12. Simpson J, Sanchez-Ovando $\mathrm{S}$, Powell $\mathrm{H}$, et al. Increased sputum FKBP51 gene expression following Azithromycin add-on therapy in asthma. Eur Respir J 2018; 52: Suppl. 62, OA1919.

13. Nelson MR, Tipney H, Painter JL, et al. The support of human genetic evidence for approved drug indications. Nat Genet 2015; 47: 856-860.

14. Chief Medical Officer. Annual Report of the Chief Medical Officer 2016: Generation Genome. Department of Health and Social Care, 2017.

15. Oelsner E, Smith B, Nguyen J, et al. Late Breaking Abstract - Associations between a COPD genetic risk score and lung structure on computed tomography (CT): SPIROMICS. Eur Respir J 2018; 52: Suppl. 62, OA2187.
16. Terzikhan N, Sun F, Verhamme F, et al. Heritability and genome-wide association study of diffusing capacity of the lung (DLCO). Eur RespirJ 2018; 52: Suppl. 62, OA2189.

17. Sugier P-E, Sarnowski C, Granell R, et al. Genome-wide interaction study of environmental tobacco smoke exposure on time-to-asthma onset in childhood. Eur Respir J 2018; 52: Suppl. 62, PA1278.

18. Wain L, Shrine N, Guyatt A, et al. A weighted genetic risk score based on 279 signals of association with lung function predicts Chronic Obstructive Pulmonary Disease. Eur Respir J 2018; 52: Suppl. 62, OA2188.

19. Bui DS, Lodge CJ, Burgess JA, et al. Childhood predictors of lung function trajectories and future COPD risk: a prospective cohort study from the first to the sixth decade of life. Lancet Respir Med 2018; 6: 535-544.

20. Belgrave DCM, Granell R, Turner SW, et al. Lung function trajectories from pre-school age to adulthood and their associations with early life factors: a retrospective analysis of three population-based birth cohort studies. Lancet Respir Med 2018; 6: 526-534.

21. Morrow LA, Wagner BD, Ingram DA, et al. Antenatal determinants of bronchopulmonary dysplasia and late respiratory disease in preterm infants. Am J Respir Crit Care Med 2017; 196: 364-374.

22. Sriram S, Schreiber MD, Msall ME, et al. Cognitive development and quality of life associated with BPD in 10-year-olds born preterm. Pediatrics 2018; 141: e20172719.

23. Ramette A, Spycher BD, Wang J, et al. Longitudinal associations between respiratory infections and asthma in young children. Am J Epidemiol 2018; 187: 1714-1720.

24. van Meel ER, den Dekker HT, Elbert NJ, et al. A population-based prospective cohort study examining the influence of early-life respiratory tract infections on school-age lung function and asthma. Thorax 2018; 73 : 167-173.

25. Loewen K, Monchka B, Mahmud SM, et al. Prenatal antibiotic exposure and childhood asthma: a population-based study. Eur Respir J 2018; 52: 1702070.

26. Dai X, Dharmage S, Abramson M, et al. GST genotypes modify the association between paracetamol use in early life and lung function at 18 years. Eur Respir J 2018; 52: Suppl. 62, OA3309.

27. Bisgaard H, Stokholm J, Chawes BL, et al. Fish oilderived fatty acids in pregnancy and wheeze and asthma in offspring. N Engl J Med 2016; 375: 2530-2539.

28. Keet CA, Keller JP, Peng RD. Long-term coarse particulate matter exposure is associated with asthma among children in medicaid. Am J Respir Crit Care Med 2018; 197: 737-746.

29. Kuiper IN, Svanes C, Abramson MJ, et al. Lung health in adulthood after childhood exposure to air pollution and greenness. Eur RespirJ 2018; 52: Suppl. 62, OA5185. 
30. Casas M, Abellan A, Garcia R, et al. Prenatal exposure to organochlorine compounds and lung function until early adulthood. Eur RespirJ 2018; 52: Suppl. 62, PA5015.

31. Cecchi L, D’Amato G, Annesi-Maesano I. External exposome and allergic respiratory and skin diseases. J Allergy Clin Immunol 2018; 141: 846-857.

32. Siroux V, Agier L, Basagaña X, et al. Early life exposome and lung function in children from the HELIX cohort. Eur Respir J 2018; 52: Suppl. 62, OA5184

33. Kjaergaard JL, Juhl CB, Rosenberg $M$, et al. Early pulmonary rehabilitation after acute exacerbation of COPD. Eur RespirJ 2018; 52: Suppl. 62, OA1618.

34. Currow D, Louw S, Mccloud $P$, et al. Regular extended release morphine for chronic breathlessness: a multi-centre double-blind RCT. Eur RespirJ 2018; 52: Suppl. 62, OA1624.

35. Bhatt S, Balte $P$, Cassano $P$, et al. Late Breaking Abstract - Optimizing FEV1/FVC threshold to predict COPD hospitalization and mortality: NHLBI pooled cohorts study. Eur RespirJ 2018; 52: Suppl. 62, PA3337.

36. Karcioğlu O, Sarinc Ulasli S, Demir AU. Investigation of relationship between four meter gait speed test and cardiopulmonary exercise test to determine exercise capacity in COPD patients. Eur Respir J 2018; 52: Suppl. 62, PA3343.
37. The burden of lung disease. In: Gibson J, Loddenkemper R, Sibille Y, et al., eds. European Lung White Book. Sheffield, European Respiratory Society, 2013; pp. 2-15.

38. Horváth I, Barry M, Brusselle $G$, et al. The European Respiratory Society's 10 Principles for Lung Health. Eur Respir 2018; 52: 1801373.

39. Secher T, Dalonneau E, Parent C, et al. Airway administration of therapeutic antibody ( $t A b)$ confers higher protection than parenteral administration in a murine model of acute lung infection. Eur Respir J 2018; 52: Suppl. 62, PA5451

40. Raghu G, Remy-Jardin M, Myers JL, et al. Diagnosis of Idiopathic Pulmonary Fibrosis. An Official ATS/ERS/JRS/ALAT Clinical Practice Guideline. Am J Respir Crit Care Med 2018; 198: e44-e68.

41. Maher TM, Stowasser S, Nishioka Y, et al. Investigating the effects of nintedanib on biomarkers of extracellular matrix turnover in patients with IPF: design of the randomised placebo-controlled INMARK(R)trial. BMJ Open Respir Res 2018; 5: e000325.

42. Kolb M, Raghu G, Wells AU, et al. Nintedanib plus sildenafil in patients with idiopathic pulmonary fibrosis. $N$ Engl J Med 2018; 379: 1722-1731. 